\title{
Efeitos de detomidina e xilazina intravenosa sobre as variáveis basais e respostas comportamentais em bovinos
}

\author{
[Effects of intravenous detomidine and xilazine on basal parameters and behavioral \\ responses in bovine] \\ G. Ribeiro ${ }^{1}$, R.G.S. Dória ${ }^{2}$, T.C. Nunes ${ }^{3}$, A.L. Gomes $^{3}$, W.A.B. Pereira ${ }^{3}$, \\ F.F. Queiroz ${ }^{3}$, A.B. Vasconcelos ${ }^{3}$ \\ ${ }^{1}$ Faculdades Metropolitanas Unidas - FMU - São Paulo, SP \\ ${ }^{2}$ Faculdade de Zootecnia e Engenharia de Alimentos - FZEA-USP - Pirassununga, SP \\ ${ }^{3}$ Universidade de Uberaba - UNIUBE - Uberaba, MG
}

\begin{abstract}
RESUMO
Avaliaram-se, durante 60 minutos, 10 bovinos após administração intravenosa de $0,1 \mathrm{mg} \cdot \mathrm{kg}^{-1}$ de xilazina ou $10 \mu \mathrm{g} . \mathrm{kg}^{-1}$ de detomidina, quanto às frequências cardíaca e respiratória, movimentos ruminais, pressão arterial média, temperatura retal e respostas comportamentais como ataxia ou decúbito, ptose palpebral, estado de alerta ou sedação e redução da altura da cabeça em relação ao solo, além da presença de salivação, micção e concentração sanguínea de glicose. Observou-se que a xilazina, via intravenosa, em bovinos, ao mesmo tempo que promove sedação mais intensa e prolongada que a detomidina, induz a uma maior quantidade de efeitos indesejáveis, como salivação e decúbito, e redução das frequências cardíaca e respiratória, da pressão arterial média, da motilidade ruminal e da temperatura, sendo estas alterações mais prolongadas. Conclui-se que a detomidina pode ser utilizada com segurança em bovinos na dose de $10 \mu \mathrm{g} . \mathrm{kg}^{-1}$, promovendo sedação e permanência do animal em posição quadrupedal.
\end{abstract}

Palavras-chave: bovino, detomidina, xilazina

\begin{abstract}
Ten bovine were evaluated after intravenous injection of $0,1 \mathrm{mg}^{\mathrm{kg}} \mathrm{kg}^{-1}$ of xylazine or $10 \mu \mathrm{g} . \mathrm{kg}^{-1}$ of detomidine during 60 minutes for heart and respiratory rate, ruminal motility, mean arterial pressure, rectal temperature and behavioral responses like ataxia or recumbency, palpebral ptoses, state of sedation or alert and head drop, besides the measurement of salivation, urination and blood glucose concentration. It was observed that intravenous xylazine in bovine promotes more intense and prolonged sedation than detomidine, and at the same time induces a larger and more prolonged quantity of unwanted side effects such as salivation, recumbency, decrease of cardiac and respiratory rate, mean arterial pressure, ruminal motility and temperature. We concluded that detomidine can be used safely in bovines at $10 \mu \mathrm{g} . \mathrm{kg}^{-1}$ dose, promoting sedation with standing position.
\end{abstract}

Keywords: bovine, detomidine, xylazine

\section{INTRODUÇÃO}

A xilazina e a detomidina são substâncias com propriedades sedativa e analgésica, altamente lipofílicas, com volume de distribuição alto, cuja meia-vida de eliminação situa-se entre 30 e 90 minutos e de depuração total entre 20 e $80 \mathrm{~mL} \cdot \mathrm{kg}^{-1} \cdot \mathrm{min}^{-1}$, classificadas farmacologicamente como agonistas dos receptores adrenérgicos do tipo $\alpha_{2}$ (agonistas $\alpha_{2}$ ) (Salonen, 1992).

A xilazina foi o primeiro agonista $\alpha_{2}$ a ser utilizado clinicamente na medicina veterinária e

Recebido em 19 de agosto de 2011

Aceito em 20 de julho de 2012

E-mail: gesiane_r@yahoo.com.br 
consagra-se como o composto mais usado para imobilizar ruminantes, exercendo efeito sedativo, analgésico e relaxante muscular. Os efeitos da xilazina resultam da ativação de adrenorreceptores $\alpha_{2}$ pré-sinápticos localizados no sistema nervoso central (SNC), o que reduz as transmissões interneuronais de norepinefrina. A xilazina, também, atua nestes mesmos receptores, localizados em tecidos periféricos, como o trato gastrintestinal, útero, rins, aparelho cardiovascular, respiratório, fígado e pâncreas, promovendo efeitos adversos, como bradicardia, bradipneia, timpanismo ruminal, poliúria, salivação e hiperglicemia (Thurmon et al., 1996). Os ruminantes são extremamente sensíveis à xilazina, sendo esta 10 a 20 vezes mais potente em ruminantes que em outras espécies (Kästner, 2006).

A detomidina é um agonistas $\alpha_{2}$ que apresenta maior especificidade aos receptores adrenérgicos, associada a uma sedação e a uma analgesia mais intensas e prolongadas, se comparada diretamente com a xilazina. Estudos têm demonstrado que, assim como com a xilazina, a profundidade e a duração da sedação e da analgesia promovidas pela detomidina são dose-dependentes (Jöcle e Hamm, 1986; Lowe e Hilfiger, 1986; Kamerling et al., 1988).

Em doses clínicas, a potência de um fármaco agonista $\alpha_{2}$ está relacionada à seletividade ao receptor adrenérgico, sendo que quanto mais potente um fármaco for, menor a dose e o volume necessários para alcançar níveis similares de sedação. A seguinte ordem de seletividade tem sido descrita: medetomidina (1620:1), detomidina (260:1), clonidina (220:1) e xilazina (160:1) (Virtanen, 1986; Scheinin et al.,1989).

Atualmente, a detomidina tem sua utilização registrada apenas para equinos, entretanto tem sido administrada clinicamente também em bovinos e em pequenos ruminantes (Singh et al., 1994; Riviere e Papich, 2009). Esteve indisponível durante muitos anos no mercado brasileiro e foi reintroduzida apenas no ano de 2008, por um laboratório de produtos veterinários, com sua apresentação farmacêutica recomendada para uso exclusivo em equinos. $\mathrm{O}$ objetivo deste trabalho foi avaliar os efeitos comportamentais, motores, cardiovasculares, respiratórios, digestórios e as variações de temperatura induzidos pela administração intravenosa de xilazina ou detomidina, em bovinos.

\section{MATERIAL E MÉTODOS}

Foram utilizados 10 bovinos, sem raça definida, com aproximadamente um ano de idade, considerados hígidos, após exames clínicos e laboratoriais, com peso corpóreo médio de $200 \pm 50 \mathrm{~kg}$, distribuídos em dois grupos experimentais: grupo xilazina $(\mathrm{GX})$, representado pelos 10 animais que receberam administração intravenosa de xilazina (Calmiun: Agener União Química, São Paulo, Brasil), e grupo detomidina (GD), correspondendo aos 10 animais que receberam administração intravenosa de detomidina (Dormiun V: Agener União Química, São Paulo, Brasil). Os mesmos animais participaram dos dois grupos experimentais, respeitando-se um intervalo de um mês entre cada procedimento.

Os animais foram submetidos a jejum alimentar de 24 horas antes do início do experimento. Após 15 minutos destinados à adaptação dos animais em tronco de contenção, as variáveis basais (T0) foram aferidas. A frequência cardíaca (FC; bpm) e os movimentos ruminais (MR; mpm) foram avaliados com auxílio de estetoscópio, e a frequência respiratória (FR; mrpm) pela observação da movimentação do gradil-costal. Utilizou-se um cateter, introduzido na artéria auricular, que fora previamente canulada, para mensurar a pressão arterial média (PAM; $\mathrm{mmHg}$, obtida pela leitura direta em manômetro. A temperatura retal $\left(\mathrm{T} ;{ }^{\circ} \mathrm{C}\right)$ foi avaliada com termômetro clínico convencional. As respostas comportamentais foram avaliadas observando-se a presença ou ausência de ataxia ou decúbito; ptose palpebral; estado de alerta ou sedação, este último caracterizado pela ausência de reação à movimentação manual na frente dos animais e ao bater de palmas e, também, pela redução da altura da cabeça em relação ao solo (H), mensurada com auxílio de régua fixada à parede do tronco, observando-se a distância do chão ao lábio inferior dos animais. Avaliou-se também a presença de salivação e micção. 
Colheu-se sangue venoso, da veia jugular, para dosagem da concentração sanguínea de glicose valor basal e 60 minutos após a administração dos fármacos. Subsequentemente, o $\mathrm{GX}$ recebeu, via intravenosa, $0,1 \mathrm{mg} \cdot \mathrm{kg}^{-1}$ de xilazina, e o GD, via intravenosa, $10 \mu \mathrm{g} \cdot \mathrm{kg}^{-1}$ de detomidina. As características foram, então, reavaliadas aos cinco (T5), 10 (T10), 20 (T20), 30 T(30), 40 $\mathrm{T}(40), 50 \mathrm{~T}(50)$ e $60 \mathrm{~T}(60)$ minutos após a administração dos fármacos.

O estudo foi realizado de maneira cega, ou seja, um pesquisador não envolvido com a colheita dos dados foi o responsável pelo abastecimento das seringas com xilazina ou detomidina, de forma que os avaliadores não tivessem conhecimento do fármaco a ser administrado.

Os dados, obtidos na fase experimental, foram submetidos à análise de variância para repetições múltiplas (RM ANOVA), para dados paramétricos - FC, FR, PAM, T, MR, H, glicose sanguínea -, seguida pelo teste SNK. Entre os grupos, nos diferentes intervalos, usou-se o testet. O dados não paramétricos - ataxia/decúbito, ptose palpebral, estado de alerta/sedação - foram submetidos a teste de Mann-Whitney. As diferenças foram consideradas estatisticamente significativas quando $\mathrm{P} \leq 0,05$.

\section{RESULTADOS}

Foi observada, no GX, redução significativa das frequências cardíaca e respiratória do T5 ao T60, em relação ao T0, e no GD, redução significativa da frequência cardíaca do T5 ao T60, e da frequência respiratória do T5 ao T40, em relação ao T0. Entre os grupos, foram observadas diferenças significativas em T5, T50 e T60, em que a frequência cardíaca do GX foi menor que a do GD. Foi observada, no GX e no GD, redução significativa dos movimentos ruminais, respectivamente, do T5 ao T50 e do T5 ao T30, em relação ao T0. No GX, foi observada redução das médias de pressão arterial média do T5 ao T50 em relação ao T0, e foi observada, no GD, elevação significativa da média de pressão arterial média, apenas no T5, em relação ao T0.
Entre os grupos, foram observadas diferenças significativas em T5, T10 e T20, em que a pressão arterial média do GX mostrou-se inferior à do GD. Não foi observada, no GX e no GD, em relação ao T0, alteração das médias de temperatura corporal durante o período experimental. Entretanto, entre os grupos, foram observadas diferenças significativas em T30, T40 e T50, em que a temperatura do GX mostrou-se inferior à do GD. No GX e no GD, os animais permaneceram sedados durante os 60 minutos de avaliação, caracterizados pela ausência de reação a movimentos manuais ou bater de palmas. No GX, foi observada redução da altura da cabeça em relação ao solo, ptose palpebral e salivação do T5 ao T60, em relação ao T0. Todos os animais do GD apresentaram ptose palpebral e salivação, significativas do T5 ao T40, em relação ao T0. Houve redução significativa da altura das cabeças no T10 em relação ao T0. No GX, os animais apresentaram ataxia do T5 ao T50, sendo que do T5 ao T20 todos os animais (100\%) permaneceram em decúbito; no T30, $80 \%$ dos animais estavam em decúbito e $20 \%$ apresentavam-se severamente atáxicos, em estação; no T40, todos os animais (100\%) apresentavam-se atáxicos, porém já permaneciam em estação; no T50, 50\% dos animais apresentavam-se atáxicos, em estação, e $50 \%$ já não apresentavam alteração da função motora, e no T60, 90\% dos animais não apresentavam alteração motora e $10 \%$ ainda encontravam-se com ataxia, em estação. Todos os animais no GD apresentaram significativa ataxia do T5 ao T60 em relação ao T0, embora tenham permanecido todo o período em estação. Entre os grupos, foram observadas diferenças significativas em T5, T10 e T20, em que a redução da altura da cabeça em relação ao solo e a ataxia apresentadas pelos animais do GX mostraram-se mais acentuadas que no GD, sendo que no T5 evidenciava-se uma maior sedação e ptose palpebral nos animais do GX em relação ao GD. No GX e no GD, a concentração de glicose sanguínea elevou-se significativamente no T60 em relação ao T0 (Tab. 1). No GX, 30\% dos animais urinaram entre T40 e T60, e no GD, entre T30 e T60, 70\% dos animais urinaram. 
Tabela 1. Valores médios e desvios-padrão $(\mathrm{X} \pm \mathrm{DP})$ da frequência cardíaca ( $\mathrm{FC}$; bpm), frequência respiratória (FR; mrpm), pressão arterial média (PAM; mmHg), movimentos ruminais (MR; mpm), temperatura $\left(\mathrm{T} ;{ }^{\circ} \mathrm{C}\right)$ e altura da cabeça em relação ao solo $(\mathrm{H} ; \mathrm{cm})$, e avaliação dos efeitos sedativo e motor (médias dos animais em estação, atáxico ou em decúbito) e concentração de glicose sanguínea (GS; $\mathrm{mg} / \mathrm{dL}$ ) obtidos em bovinos tranquilizados com xilazina (GX) ou detomidina (GD), via intravenosa

\begin{tabular}{|c|c|c|c|c|c|c|c|c|c|c|}
\hline & Tempo & & T0 & T5 & T10 & T20 & T30 & $\mathrm{T} 40$ & T50 & T60 \\
\hline & GX & $\bar{X}$ & 62 & $45^{\# *}$ & $45^{\#}$ & $45^{\#}$ & $47^{\#}$ & $49^{\#}$ & $49^{\#} *$ & $50^{\# *}$ \\
\hline $\mathrm{FC}$ & & $\pm \mathrm{DP}$ & 3,3 & 4,6 & 4,5 & 4,1 & 6,2 & 5,7 & 6,4 & 6,4 \\
\hline \multirow[t]{3}{*}{ (bpm) } & GD & $X$ & 65 & $49^{\#}$ & $49^{\#}$ & $51^{\#}$ & $53^{\#}$ & $54^{\#}$ & $56^{\#}$ & $57^{\#}$ \\
\hline & & $\pm \mathrm{DP}$ & 8,5 & 4,0 & 5,5 & 7,3 & 8,0 & 7,8 & 7,7 & 7,7 \\
\hline & GX & $\mathrm{X}$ & 40 & $17^{\#}$ & $15^{\#}$ & $12^{\#}$ & $12^{\#}$ & $14^{\#}$ & $16^{\#}$ & $22^{\#}$ \\
\hline FR & & $\pm \mathrm{DP}$ & 12,8 & 5,3 & 5,0 & 3,8 & 3,5 & 3,8 & 5,8 & 6,3 \\
\hline \multirow[t]{3}{*}{ (mrpm) } & GD & X & 32 & $21^{\#}$ & $17^{\#}$ & $15^{\#}$ & $17^{\#}$ & $19^{\#}$ & 24 & 27 \\
\hline & & $\pm \mathrm{DP}$ & 8,7 & 12,0 & 10,2 & 10,0 & 10,0 & 6,9 & 10,4 & 10,9 \\
\hline & GX & $\mathrm{X}$ & 122 & $81^{\# *}$ & $78^{\# *}$ & $84^{\# *}$ & $110^{\#}$ & $113^{\#}$ & $115^{\#}$ & 119 \\
\hline PAM & & $\pm \mathrm{DP}$ & 10,5 & 13 & 12,0 & 15,5 & 16,0 & 11,0 & 10,2 & 9,2 \\
\hline \multirow[t]{3}{*}{$(\mathrm{mmHg})$} & GD & $X$ & 114 & $132^{\#}$ & 118 & 111 & 112 & 114 & 112 & 116 \\
\hline & & $\pm \mathrm{DP}$ & 9,2 & 19,2 & 17,4 & 15,4 & 19,7 & 13,2 & 10,3 & 11,8 \\
\hline & GX & $\mathrm{X}$ & 5 & $0,4^{\#}$ & $0,2^{\#}$ & $0,5^{\#}$ & $1,5^{\#}$ & $2,5^{\#}$ & $3,4^{\#}$ & 5 \\
\hline \multirow{4}{*}{$\begin{array}{c}\text { MR } \\
(\mathrm{mpm})\end{array}$} & & $\pm \mathrm{DP}$ & 1,1 & 0,5 & 0,4 & 0,7 & 1,0 & 1,1 & 1,6 & 0,6 \\
\hline & GD & $X$ & 4,5 & $0,9^{\#}$ & $0,8^{\#}$ & $0,6^{\#}$ & $2,1^{\#}$ & 3,6 & 4 & 4,1 \\
\hline & & $\pm \mathrm{DP}$ & 1,5 & 1,4 & 0,8 & 0,8 & 1,6 & 1,9 & 1,3 & 1,3 \\
\hline & GX & $\mathrm{X}$ & 39,1 & 39,1 & 39,1 & 39,1 & $39,1 *$ & $39,2 *$ & $39,1 *$ & 39,2 \\
\hline \multirow{4}{*}{$\begin{array}{c}\mathrm{T} \\
\left({ }^{\circ} \mathrm{C}\right)\end{array}$} & & $\pm \mathrm{DP}$ & 0,6 & 0,6 & 0,5 & 0,5 & 0,6 & 0,5 & 0,6 & 0,6 \\
\hline & GD & $X$ & 39,3 & 39,4 & 39,5 & 39,5 & 39,6 & 39,6 & 39,6 & 39,3 \\
\hline & & $\pm \mathrm{DP}$ & 0,4 & 0,6 & 0,4 & 0,4 & 0,4 & 0,2 & 0,2 & 0,5 \\
\hline & GX & $\mathrm{X}$ & 66,5 & $0,5^{\#} *$ & $0,3^{\#} *$ & $0,5^{\#} *$ & $40,3^{\#}$ & $55,7^{\#}$ & $55,5^{\#}$ & $56,4^{\#}$ \\
\hline \multirow{3}{*}{$\begin{array}{c}\mathrm{H} \\
(\mathrm{cm})\end{array}$} & & $\pm \mathrm{DP}$ & 9,0 & 1,3 & 0,7 & 1,0 & 22,8 & 10,2 & 6,7 & 7,1 \\
\hline & GD & $\mathrm{X}$ & 59,7 & 52,6 & $46,8^{\#}$ & 54,2 & 53,7 & 57 & 59,3 & 59,6 \\
\hline & & $\pm \mathrm{DP}$ & 10,6 & 5,6 & 14,4 & 7,6 & 8,3 & 8,7 & 9,0 & 8,6 \\
\hline \multirow{2}{*}{$\begin{array}{l}\text { Efeito } \\
\text { motor }\end{array}$} & GX & & $E$ & $\mathrm{D}^{\# *}$ & $\mathrm{D}^{\# *}$ & $\mathrm{D}^{\#_{*}}$ & $\mathrm{~A}^{\#}$ & $\mathrm{~A}^{\#}$ & $\mathrm{~A}^{\#}$ & $\mathrm{E}$ \\
\hline & GD & & E & $\mathrm{A}^{\#}$ & $\mathrm{~A}^{\#}$ & $\mathrm{~A}^{\#}$ & $\mathrm{~A}^{\#}$ & $\mathrm{~A}^{\#}$ & $\mathrm{~A}^{\#}$ & $\mathrm{~A}^{\#}$ \\
\hline \multirow{2}{*}{$\begin{array}{c}\text { Efeito } \\
\text { sedativo }\end{array}$} & GX & & - & $+{ }^{\# *}$ & $+{ }^{\#}$ & $+\#$ & $+\#$ & $+\#$ & $+\#$ & $+\#$ \\
\hline & GD & & - & $+{ }^{\#}$ & $+{ }^{\#}$ & $+{ }^{\#}$ & $+{ }^{\#}$ & $+{ }^{\#}$ & $+{ }^{\#}$ & $+{ }^{\#}$ \\
\hline \multirow{2}{*}{$\begin{array}{c}\text { Ptose } \\
\text { palpebral }\end{array}$} & GX & & - & $+{ }^{\# *}$ & $+{ }^{\#}$ & $+\#$ & $+{ }^{\#}$ & $+{ }^{\#}$ & $+{ }^{\#}$ & $+{ }^{\#}$ \\
\hline & GD & & - & $+{ }^{\#}$ & $+{ }^{\#}$ & $+{ }^{\#}$ & $+{ }^{\#}$ & $+{ }^{\#}$ & - & - \\
\hline \multirow[t]{2}{*}{ Salivação } & GX & & - & $+{ }^{\# *}$ & $+\#$ & $++^{\#}$ & $+\#$ & $+\#$ & $+{ }^{\#}$ & $+{ }^{\#}$ \\
\hline & GD & & - & $+{ }^{\#}$ & $+{ }^{\#}$ & $+{ }^{\#}$ & $+{ }^{\#}$ & $+{ }^{\#}$ & - & - \\
\hline \multirow{4}{*}{$\begin{array}{c}\text { GS } \\
(\mathrm{mg} / \mathrm{dL})\end{array}$} & GX & $\mathrm{X}$ & 80,7 & & & & & & & $143,7^{\#}$ \\
\hline & & $\pm \mathrm{DP}$ & 20,3 & & & & & & & 19,5 \\
\hline & GD & $X$ & 78,2 & & & & & & & $160,4^{\#}$ \\
\hline & & $\pm \mathrm{DP}$ & 6,7 & & & & & & & 34,3 \\
\hline
\end{tabular}

A: ataxia; D: decúbito; E: estação, ausência de ataxia; +: presente; -: ausente.

\# diferença significativa, quando comparada a T0 (teste de SNK, $\mathrm{P} \leq 0,05$ ).

* diferença significativa entre os grupos (teste " $t$ " ou Mann-Whitney, $\mathrm{P} \leq 0,05$ ).

\section{DISCUSSÃO}

Os agonistas $\alpha_{2}$ são conhecidos por mediar uma variedade de efeitos, incluindo sedação, analgesia, hipertensão inicial seguida de hipotensão, bradicardia, bradipneia e hipotermia (Yamashita et al., 2000; Lin e Riddell, 2003;
Read, 2003). No tocante à avaliação cardiorrespiratória dos bovinos após a administração intravenosa de xilazina e detomidina, neste estudo houve redução da frequência cardíaca, durante os 60 minutos de avaliação, nos dois grupos experimentais, e redução da frequência respiratória, durante 60 
minutos, no grupo xilazina, e 40 minutos no grupo detomidina, embora os valores tenham permanecido dentro dos considerados fisiológicos. A pressão arterial média, durante os 60 minutos da avaliação experimental, apresentou-se reduzida no grupo xilazina; no grupo detomidina, apresentou uma elevação aos cinco minutos após a administração do fármaco, tendo permanecido dentro dos valores considerados fisiológicos nos demais tempos experimentais. Sendo assim, ficou demonstrado com este estudo que a administração intravenosa de xilazina ou detomidina promove alterações cardiovasculares e respiratórias, a partir de cinco minutos pós-administração, sendo o efeito depressivo, mais intenso e duradouro, quando se administra xilazina, corroborando com Lin e Riddell (2003).

A redução da motilidade ruminal foi fato observado nos dois grupos experimentais, sendo mais prolongada no grupo em que se administrou xilazina (50 minutos). Estudo realizado por Ruckebusch e Allal (1987) demonstrou que os agonistas $\alpha_{2}$ inibem a contração ruminal primária que está associada à contração reticular e suprime a contração ruminal secundária. Sugerese, então, uma inibição da atividade motora cíclica do retículo-rúmen mediada pelos $\alpha_{2}$ adrenorreceptores.

Neste estudo, pode ser observado que os dois agonistas $\alpha_{2}$, via intravenosa, promovem sedação em bovinos, a qual permanece por um período de até 60 minutos e é caracterizada por ausência de resposta a estímulos manuais e bater de palmas, redução da altura da cabeça em relação ao solo e ptose palpebral. Da mesma forma, estudo realizado por Lin e Riddell (2003) demonstrou maior tempo de sedação dos bovinos que receberam xilazina (49 minutos) em relação aos que receberam detomidina (47 minutos), sendo que todos os animais também demonstraram bradicardia e bradipneia significante, além de ptose palpebral e salivação.

Estes fármacos promoveram ataxia, sendo que apenas a xilazina induziu decúbito em $100 \%$ dos animais, durante 20 minutos, a qual permaneceu por um período de 30 minutos em $80 \%$ dos bovinos. Segundo Waterman et al. (1987), uma dose de detomidina de $10 \mu \mathrm{g} . \mathrm{kg}^{-1}$, via intravenosa, em carneiros adultos, induz profunda sedação sem decúbito, sendo que apenas doses mais altas $\left(30-140 \mu \mathrm{g} \cdot \mathrm{kg}^{-1}\right)$ induzem, via intravenosa ou intramuscular, sedação profunda e relaxamento muscular com ataxia severa e decúbito (doses maiores que 60 $\mu \mathrm{g} . \mathrm{kg}^{-1}$ ) (Komar, 1989; Short, 1992; Singh et al., 1994). Tal fato pode também ser observado neste estudo, já que $10 \mu \mathrm{g} \cdot \mathrm{kg}^{-1}$ de detomidina, via intravenosa, não promoveram decúbito em nenhum dos bovinos.

A hipersiália apresentada por bovinos após a administração de agonistas $\alpha_{2}$, referida por alguns autores por se tratar apenas de uma diminuição do reflexo de deglutição, com acumulação de saliva na cavidade bucal (Plumb, 1995; Thurmon et al., 1999), foi evidenciada neste estudo em todos os animais, após a administração da xilazina e da detomidina, sendo mais prolongada após a administração da primeira (60 minutos).

$\mathrm{O}$ efeito diurético dos agonistas $\alpha_{2}$, que ocorre, provavelmente, devido à diminuição da produção de vasopressina (Plumb, 1995; Thurmon et al., 1999), embora tenha sido observado nos dois grupos durante a colheita dos dados experimentais, foi mais evidente no grupo detomidina, o qual, também, apresentou maior elevação da glicemia após 60 minutos da administração do fármaco, concordando com o estudo realizado por Peshin et al. (1991), que demonstrou, em bezerros, após $10 \mu \mathrm{g} \cdot \mathrm{kg}^{-1}$ de detomidina, sedação por 30 a 45 minutos e hiperglicemia. Embora o mecanismo de ação que provoca hiperglicemia ainda não tenha sido completamente esclarecido, ela pode ocorrer em consequência de um aumento na produção de glicose pelo fígado e da diminuição da secreção pancreática de insulina (estimulação dos receptores $\alpha_{2}$ ), reduzindo a utilização de glicose sanguínea pelos tecidos periféricos (Lima et al., 2001).

Este estudo demonstra que a xilazina, via intravenosa, em bovinos, ao mesmo tempo que promove uma sedação mais intensa e prolongada que a detomidina, induz a uma maior quantidade de efeitos indesejáveis, como salivação, decúbito, redução das frequências cardíaca, respiratória, pressão arterial média, motilidade ruminal e temperatura, sendo estas alterações, da mesma forma, mais prolongadas. Estes resultados se contrapõem à literatura, que salienta o fato de que, embora a detomidina seja 
um fármaco mais seletivo aos receptores adrenérgicos do tipo $\alpha_{2}$ do que a xilazina, as diferenças farmacodinâmicas, em doses equipotentes, são mínimas, exceto pela duração da ação dos fármacos (England e Clarke, 1996; Bueno et al., 1999; Riviere e Papich, 2009).

Sugere-se, então, o desenvolvimento de estudo que busque a dose equipotente entre a xilazina e a detomidina para bovinos. Supõe-se, com este estudo, que exista uma diferença entre espécies em relação aos efeitos da xilazina e detomidina, uma vez que, via intravenosa, a dose de $10 \mu \mathrm{g} . \mathrm{kg}$ ${ }^{1}$ promove o mesmo grau e duração de sedação que $0,1 \mathrm{mg} / \mathrm{kg}$ de xilazina, sem causar decúbito, apresentando efeitos adversos menos intensos e por um período de tempo mais curto, o que torna a detomidina um fármaco conveniente para a realização de procedimentos cirúrgicos com o animal sedado e em estação.

\section{CONCLUSÕES}

A detomidina pode ser utilizada com segurança em bovinos na dose de $10 \mu \mathrm{g} . \mathrm{kg}^{-1}$, promovendo sedação e permanência do animal em posição quadrupedal.

\section{REFERÊNCIAS}

BUENO, A.C.; SEAHORN, J.C.; SEAHORN, T.L. et al. Cardiopulmonary and sedative effects of intravenous administration of low doses of medetomidine and xylazine to adult horses. Am. J. Vet. Res., v.60, p.1371-1376, 1999.

ENGLAND, G.C.W.; CLARKE, K.W. Alpha-2 adrenoceptor agonists in the horse, a review. Br. Vet J., v.152, p.641-657, 1996.

JÖCHLE, W.; HAMM, D. Sedation and analgesia with domosedan (detomidine hydrochloride) in horses - dose response studies on efficacy and its duration. Acta Vet. Scand., v.82, p.69-85, 1986.

KAMERLING, S.G.; CRAVENS, W.M.T.; BAGWELL, C.A. Objective assessment of detomidine-induced analgesia and sedation in the horse. Eur. J. Pharmacol., v.151, p.1-8, 1988.

KÄSTNER, S.B.R. A2-agonists in sheep: a review. Vet. Anaesth. Analg., v.33, p.79-96, 2006.

KOMAR, E. Detomidine as sedative in sheep. Folia Vet., v.33, p.9-17, 1989.
LIMA, M.S.; MALTA, M.; LAMAS, L. Comparação dos efeitos hiperglicemiantes da xilazina em novilhas Frísia e novilhas Mertolengas. Rev. Port. Ciên. Vet., v.96, p.149-152, 2001.

LIN, H.C.; RIDDELL, M.G. Preliminary study of the effects of xylazine or detomidine with or without butorphanol for standing sedation in dairy cattle. Vet. Ther., v.4, p.285-291, 2003.

LOWE, J.E.; HILFIGER, J. Analgesic and sedative effects of detomidine compared to xylazine in a colic model using IV and IM routes of administration. Acta Vet. Scand., v.82, p.85-95, 1986.

PESHIN, P.K.; SINGH, A.P.; SINGH J. et al. Sedative effect of detomidine in infant calves. Acta Vet. Hung., v.39, p.102-107, 1991.

PLUMB, D.C. Veterinary Drug Handbook. 2nd ed. Ames: Iowa State University Press, 1995. 722p.

READ, M.R. A review of alpha-2 adrenoreceptor agonists and the development of hypoxemia in domestic and wild ruminants. J. Zoo. Wildl. Med., v.34, p.134-138, 2003.

RIVIERE, J.E.; PAPICH, M.G. Sedative agents: tranquilizers, alpha-2 agonists, and related agents. In: Veterinary Pharmacology and Therapeutics. 9 ed. New York: John Wiley \& Sons, 2009. p.348-349.

RUCKEBUSCH, Y.; ALLAL, C. Depression of reticulo-ruminal motor functions through the stimulation of alpha-2 adrenoceptor. J. Vet. Pharmacol. Ther., v.10, p.1-10, 1987.

SALONEN, J.S. Chemistry and pharmacokinetics of the $\alpha 2$-adrenoceptor agonists. In: SHORT, C.E.; POZNAK, A.V. Animal Pain. New York: Churchill Livingstone, 1992, p.191-200.

SCHEININ, H.; VIRTANEN, A.; MacDONALD, E. et al. Medetomidine - a novel alpha-2 adrenoceptor agonist: a review of its pharmacodynamic effect. Prog. Neuropsychopharmacol. Biol. Psychiatry, v.13, p.635651,1989

SHORT, C.E. Alpha-2 agents in animals - sedation, analgesia and anaesthesia. California: Veterinary Practice Publishing Company \& Brillig Hill Inc, Santa Barbara, 1992. p.8-19.

SINGH, J.; SINGH, A.P.; PEECHIN, P. Evaluation of detomidine as a sedative in sheep. Indian J. Anim. Sci., v.64, p.237-238, 1994.

THURMON, J.C.; SARR, R.; DENHART, J.W. Xylazine sedation antagonized with Tolazoline. Compendium of Continuing Education (Food Animal), v.1, p.11-20, 1999. 
Efeitos de detomidina...

THURMON, J.C.; TRANQUILLI, W.J.; BENSON, G.J. Preanesthetics and anesthetic adjuncts. In: Lumb \& Jones' Veterinary Anesthesia. 3nd ed, Baltimore: Williams \& Wilkins, 1996. p.183-209.

VIRTANEN, R. Pharmacology of detomidine and other alpha-2 adrenoceptor agonists in the brain. Acta Vet. Scand., v.82, p.35-46, 1986.
WATERMAN, A.E.; NOLAN, A.; LIVINGSTON, A.

Influence of idazoxan on the respiratory blood gas changes influenced by alpha-2 adrenoceptor agonist drugs unconscious sheep. Vet. Rec., v.121, p.105-107, 1987.

YAMASHITA, K.; TSUBAKISHITA, $\quad$ S.; FUTAOKA, S. et al. Cardiovascular effects of medetomidine, detomidine and xylazine in horses. $J$. Vet. Med. Sci., v.62, p.1025-1032, 2000. 